\title{
The Trade-off Between Transceiver Capacity and Symbol Rate
}

\author{
L. Galdino, D. Lavery, Z. Liu, K. Balakier, E. Sillekens, D. Elson, G. Saavedra, R. I. Killey \\ and P. Bayvel \\ Optical Networks Group, Dept. of Electronic \& Electrical Engineering, University College London, UK \\ l.galdino@ucl.ac.uk
}

\begin{abstract}
The achievable throughput using high symbol rate, high order QAM is investigated for the current generation of CMOS-based DAC/ADC. The optimum symbol rate and modulation format is found to be $80 \mathrm{GBd}$ DP-256QAM, with an $800 \mathrm{~Gb} / \mathrm{s}$ net data rate.
\end{abstract}

OCIS codes: (060.0060) Fiber optics and optical communications; (060.2360) Fiber optics links and subsystems

\section{Introduction}

A key goal when designing a new optical transmission system is to increase the amount of data sent for a given cost. One way to reduce the cost-per-bit is to increase the channel symbol rate, which will maximise the amount of information sent over one channel and reduce the number of transceivers for a given transmission bandwidth.

Currently, the upper limit on the available signal-to-noise ratio (SNR), and therefore channel highest achievable information rate (AIR) in a coherent optical transmission system, in the absence of fiber nonlinearity, is bounded by the transceiver subsystems, such as digital-to-analog converter (DAC) and analog to digital converter (ADC). The SNR of an ideal DAC / ADC is defined by the number of bits $(\mathrm{N})$ which sets the quantization noise floor of the device [1]. In practical converters, other distortion sources will add to this noise floor, leading to an effective number of bits (ENOB) smaller than $\mathrm{N}$ bits, resulting in a lower SNR. Additionally, mainly due to clock jitter, the ENOB is a function of frequency, and is reduced at high frequencies, which consequently diminishes the SNR as the channel symbol rate is increased. An impressive symbol rate of $100 \mathrm{GBd}$ was demonstrated in [2], achieving $1.2 \mathrm{~Tb} / \mathrm{s}$ channel line rate and $1 \mathrm{~Tb} / \mathrm{s}$ after $300 \mathrm{~km}$. In [3], an AIR of $8.3 \mathrm{~b} / \mathrm{sym}$ and $9.35 \mathrm{~b} / \mathrm{sym}$ (with probabilistic shaping) for a channel symbol rate of $66 \mathrm{GBd}$ was demonstrated, achieving $680 \mathrm{~Gb} / \mathrm{s}$ after transmission over $400 \mathrm{~km}$. Both demonstrations used an arbitrary-waveform generator (AWG) based on high-speed SiGe DACs. Todays commercial systems are deployed using CMOS-based DAC / ADC with typical symbol rates on the order of $35 \mathrm{GBd}$.

In this paper, we investigate the optimum channel symbol rate using commercial off-the-shelf $92 \mathrm{GS} / \mathrm{s}$ DACs with CMOS technology. The trade-off between transceiver symbol rate and channel SNR for a given transceiver system is also studied, and its implications on the overall system capacity for a given bandwidth is analyzed.

\section{Experimental Setup}

The experimental setup used in this work is illustrated in Fig. 1. The multi-level drive signals required for mQAM, where $\mathrm{m}=4,16,64,256$, were generated offline and digitally filtered using a root raised cosine (RRC) filter and the channel symbol rate was varied from $15 \mathrm{GBd}$ to $90 \mathrm{GBd}$ in increments of $5 \mathrm{GBd}$. Digital preemphasis was applied to the signal to compensate for the frequency response of the transmitter components.

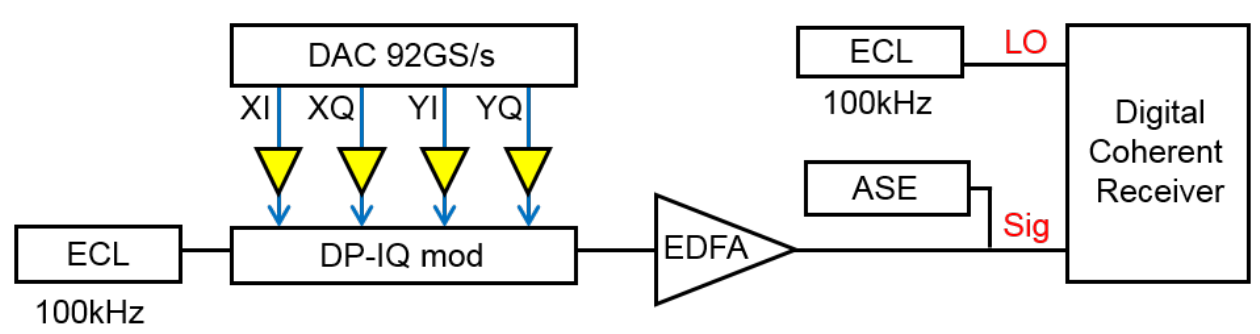

Fig. 1. Nyquist-shaped DP-mQAM experimental setup. 

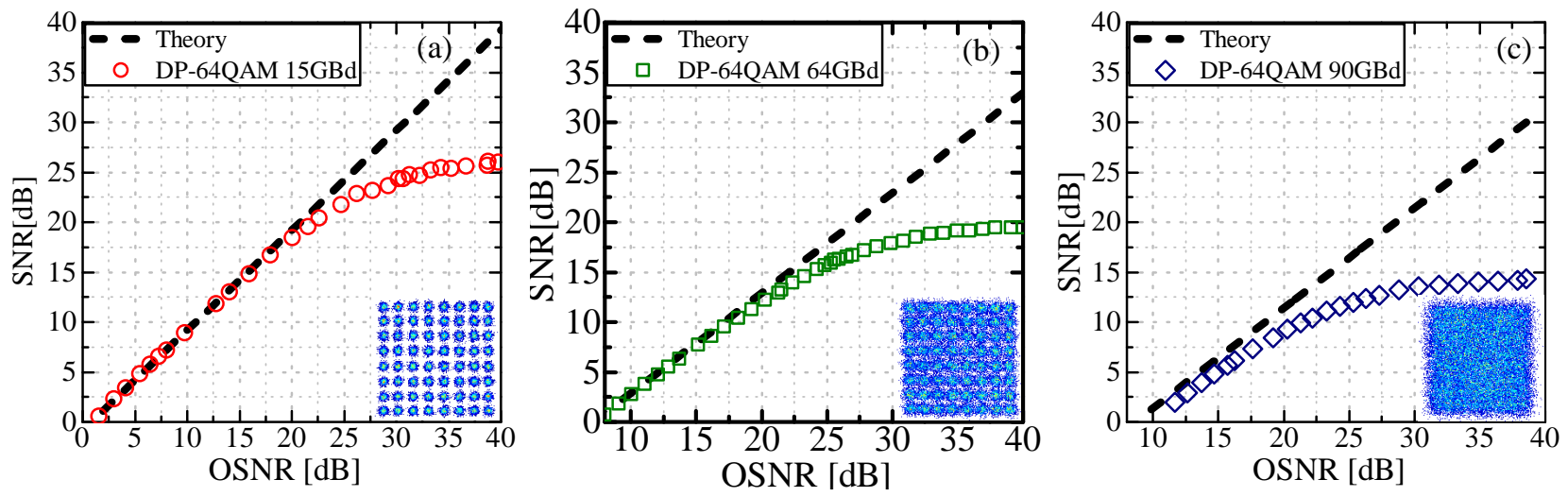

Fig. 2. Measurements of received SNR as a function of OSNR for (a) 15 GBd (b) 64 GBd and (c) 90 GBd DP-64QAM. Inset: constellation diagram from the highest SNR.

The resulting in-phase (I) and quadrature (Q) signals for each polarisation were output using four CMOS-based DACs with typical $3 \mathrm{~dB}$ analog bandwidth at $32 \mathrm{GHz}$, operating at $92 \mathrm{GSa} / \mathrm{s}$ and subsequently amplified using four linear amplifiers with $55 \mathrm{GHz}$ electrical bandwidth and typical noise figure of $6 \mathrm{~dB}$, before being applied to the Oclaro high bandwidth dual-polarisation (DP) IQ-modulator. The output of an external cavity laser (ECL) with a $100 \mathrm{kHz}$ linewidth was passed directly into the modulator before being optically amplified to form Nyquist shaped DP-mQAM optical carrier. The DP-mQAM signal was passed into the signal port of the digital coherent receiver, comprised by a $90^{\circ}$ optical hybrid, followed by $70 \mathrm{GHz}$ balanced photodiodes and a real-time digital sampling oscilloscope with a sampling rate of $160 \mathrm{GS} / \mathrm{s}(63 \mathrm{GHz}$ electrical bandwidth). The local oscillator (LO) laser was a $100 \mathrm{kHz}$ linewidth ECL. Amplified spontaneous emission noise was added to the signal to vary the received optical signal-to-noise ratio (OSNR).

The digital signal processing (DSP) was applied off-line. The DP-mQAM signal was initially compensated for receiver skew and the different responsivities of the balanced photodiodes. Each polarisation was resampled to $2 \mathrm{Sa} / \mathrm{sym}$ before matched RRC filtering. A blind 21-tap radially directed equaliser (RDE) was used to equalise the signal and to undo polarisation rotations, with the constant modulus algorithm (CMA) equaliser used for pre-convergence. The frequency offset (FO) was subsequently removed prior to blind carrier phase estimation (CPE). The total received SNR was evaluated as the ratio between the variance of the transmitted symbols $E\left[|X|^{2}\right]$ and the variance of the noise $\sigma^{2}$, where $\sigma^{2}=E\left[|X-Y|^{2}\right]$ and $Y$ represents the received symbols. The mutual information or achievable information rate (AIR) was estimated from the received data via Monte Carlo integration and provides an upper bound on the performance for any coded system based on DP-mQAM signal.

\section{Results}

Fig. 2 shows the received SNR as a function of OSNR for DP-64QAM signal with a symbol rate of (a) 15 GBd (b) $64 \mathrm{GBd}$ and (c) $90 \mathrm{GBd}$. The OSNR was recorded by adding ASE noise to the signal. The theoretical calculation of OSNR $=$ SNR $+10 \log _{10}\left(R_{S}=B\right)$, where $R_{S}$ is the symbol rate and $B$ is the noise bandwidth, is shown to provide a performance reference relative to the experimental results. If there was no practical SNR limit within the transceiver, the measured data points would follow that of the theoretical curve. However, it is evident that there is a saturation in the highest achievable SNR, and it diminishes as the channel symbol rate is increased. For instance, SNR limits of 27, 19 and $14.5 \mathrm{~dB}$ were measured for the 15,64 and $90 \mathrm{GBd}$ signal, respectively. This practical SNR limit, dominated by the effective number of bits (ENOB) of DACs and ADCs, noise figure of the drive amplifiers and DSP implementation, places an upper limit on the largest achievable information rate (AIR) for this experimental transceiver. Therefore the aim is to understand the degradation in SNR and achievable information rate (AIR) as the channel symbol rate increases.

Fig. 3 illustrates the back-to-back experimentally measured of (a) received SNR and (b) AIR, without ASE noise loading, as a function of channel symbol rate, over both polarizations of the mQAM signal. The SNR and the AIR were recorded for 16 different channel symbol rates; from $15 \mathrm{GBd}$ to up to $90 \mathrm{GBd}$. As illustrated in Fig. 3(a), for each channel symbol rate, a small variation of the received SNR was measured between the modulation formats. For a channel with $25 \mathrm{GBd}$, the received SNRs for DP-4QAM and DP-256QAM were $26.3 \mathrm{~dB}$ and $25 \mathrm{~dB}$ respectively; $1.3 \mathrm{~dB}$ lower received SNR for DP-256QAM. For a 90 GBd channel, this difference in received SNR was only $0.2 \mathrm{~dB}(14.5 \mathrm{~dB}$ for DP-4QAM and 14.3 dB from DP-256QAM). This small difference in SNR at higher symbol rate may be due to the system performance being dominated by the receiver noise.

Fig. 3(b) shows the AIR as a function of channel symbol rate for all four formats. DP-256QAM recorded the highest 

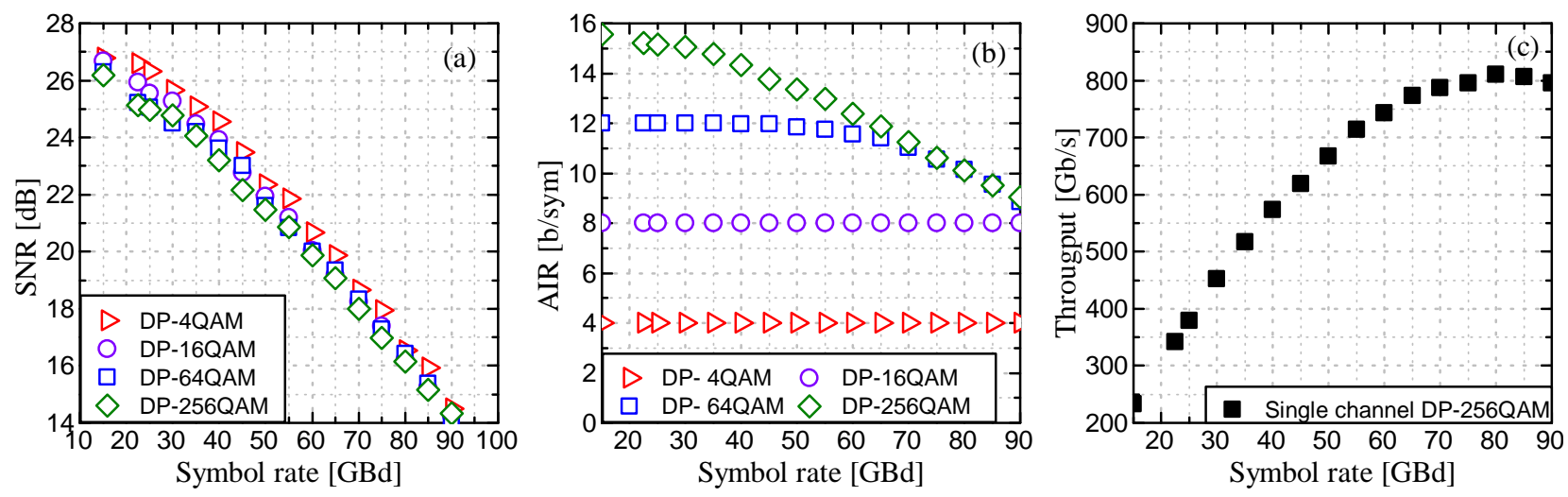

Fig. 3. DP-mQAM back-to-back measurements without ASE noise loading of (a) received SNR (b) mutual information (c) data throughput for different channel symbol rate; from $15 \mathrm{GBd}$ to $90 \mathrm{GBd}$.

achievable information rate for a channel symbol rate up to $70 \mathrm{GBd}$. For symbol rates between $70 \mathrm{GBd}$ and $90 \mathrm{GBd}$ either DP-256QAM or DP-64QAM provided the same AIR. The AIR was $15.6 \mathrm{~b} / \mathrm{sym}$ for a $15 \mathrm{GBd}$ channel, decreasing to $13.8 \mathrm{~b} / \mathrm{sym}$ for a $45 \mathrm{GBd}$ channel, and further decreasing to $9 \mathrm{~b} / \mathrm{sym}$ as the channel symbol rate approached $90 \mathrm{GBd}$. This system has enough SNR margin to maintain the highest AIR of $4 \mathrm{~b} / \mathrm{sym}$ from DP-4QAM and $8 \mathrm{~b} / \mathrm{sym}$ from DP16QAM across all channel symbol rates. For DP-64QAM an AIR of $12 \mathrm{~b} / \mathrm{sym}$ was maintained for symbol rates of up to $45 \mathrm{GBd}$ and it dropped to $9 \mathrm{~b}$ /sym for a $90 \mathrm{GBd}$ channel. Due to system SNR margin, if higher order QAM such as DP-1024QAM was generated with this transceiver setup, the ultimate performance in terms of bits per symbol would be higher than the DP-256QAM for channel symbol rates of up to $30 \mathrm{GBd}$.

Fig. 3(c) illustrates the net data throughput for different channel symbol rates. This was obtained by multiplying the corresponding DP-256QAM MI of each channel symbol rate (as shown in Fig. 3(b)) by the channel bandwidth. As expected, there was an increase in channel throughput as the channel symbol rate was increased; from $200 \mathrm{~Gb} / \mathrm{s}$ to $810 \mathrm{~Gb} / \mathrm{s}$ for channel symbol rates of $15 \mathrm{GBd}$ and $80 \mathrm{GBd}$, respectively. However there is no further benefit of increasing the channel symbol rate beyond $80 \mathrm{GBd}$, due to a sharp decrease in the transceiver SNR towards $90 \mathrm{GBd}$ which achieves a net data rate of $795 \mathrm{~Gb} / \mathrm{s}$.

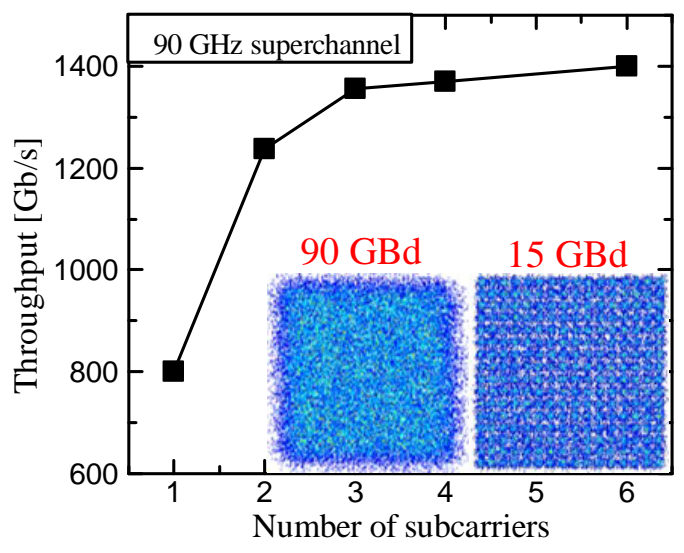

Fig. 4. Throughput versus number of subcarriers
Finally, we analyzed the overall data throughput for DP256QAM with fixed channel bandwidth. The impact of varying subcarrier bandwidth on achievable data rate for a transceiver-noise limited system was investigated. As illustrated in Fig. 4, the channel bandwidth was fixed at $90 \mathrm{GHz}$ and the performance of a $90 \mathrm{GBd}$ Nyquist-spaced superchannel with different numbers of subcarriers (such as $6 \times 15 \mathrm{GBd}, 4 \times 22.5 \mathrm{GBd}, 3 \times 30 \mathrm{GBd}, 2 \times 45 \mathrm{GBd}$ and $1 \times 90 \mathrm{GBd}$ ) was investigated. Inset are the 256QAM constelation for $90 \mathrm{GBd}$ and $15 \mathrm{GBd}$ channels. A sharp drop in the overall channel throughput was found for $1 \times 90 \mathrm{GBd}$. For instance, the gross data throughput for $6 \times 15$ GBd was $1.4 \mathrm{~Tb} / \mathrm{s}$, decreasing to $1.25 \mathrm{~Tb} / \mathrm{s}$ for $2 \times 45 \mathrm{GBd}$ with a further decrease to $795 \mathrm{~Gb} / \mathrm{s}$ for $1 \times 90 \mathrm{GBd}$ channel.

\section{Conclusion}

For a fixed superchannel bandwidth of $90 \mathrm{GHz}$ a reduction of $57 \%$ in the net channel rate was found for $1 \times 90 \mathrm{GBd}$ compared to $6 \times 15 \mathrm{GBd}$ subcarriers Nyquist-spaced. An optimum symbol rate $80 \mathrm{GBd}$ was found for a CMOS-based $\mathrm{DAC} / \mathrm{ADC}$.

This work has been supported by UK EPSRC UNLOC project. The authors would like to thank Oclaro for the High Bandwidth Polarization Multiplexed Quad Mach-Zehnder Modulator

\section{References}

[1] C. Laperle et al., "Advances in high-speed DACs, ADCs, and DSP for optical coherent transceivers," J. Lightw. Technol. 34(4), (2014).

[2] K. Schuh et al., "Single carrier 1.2Tbit/s transmission over 300km with PM-64QAM at 100GBaud," Proc. OFC, PDP Th5B.5 (2017).

[3] R. Maher et al., "Constellation shaped 66GBd DP-1024QAM Transceiver with 400km transmission over standard SMF," Proc. ECOC,(2017). 